\title{
Chapter
}

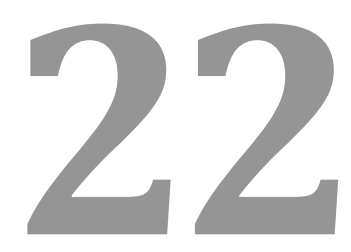

\section{MECHANICAL GUIDANCE OF CELL MIGRATION}

Ilaria Elena Palamà1, Stefania D'Amone ${ }^{1}$, Barbara Cortese ${ }^{2 *}$

${ }^{1}$ Nanotechnology Institute, CNR-NANOTEC, via Arnesano, Lecce, Italy

${ }^{2}$ Nanotechnology Institute, CNR-NANOTEC, University La Sapienza, P.zle Aldo Moro, Roma, Italy 


\section{Contents}

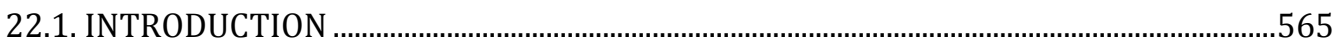

22.2. THEORETICAL MECHANISMS OF DUROTAXIS …...............................................................566

22.3. DIVERSITY OF MECHANOTACTIC CELL BEHAVIOUR …...................................................567

22.4. INFLUENCE OF ECM STIFFNESS ON CELLS........................................................................568

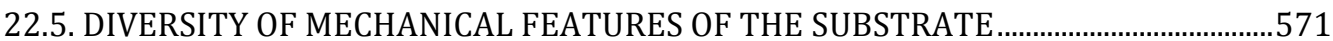

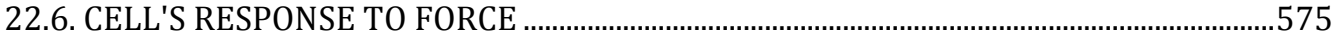

22.7. OVERRIDING ROLE OF CHEMOTAXIS OR MECHANOTAXIS? ..............................................577

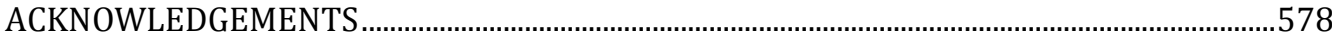

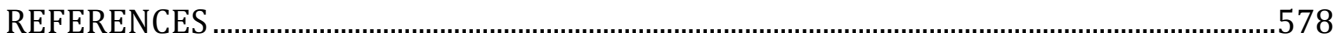




\subsection{INTRODUCTION}

Cell development and morphogenesis, wound healing or tumour invasion are all biological processes which require cells to be able to migrate following directional cues. In recent years, much attention has been paid on cell signalling and movement as regulated by many kinds of stimuli such as chemotaxis [1,2], galvanotaxis [3] and extracellular matrix (ECM) compliance/stiffness and topology [4-8] as shown in Figure 1.

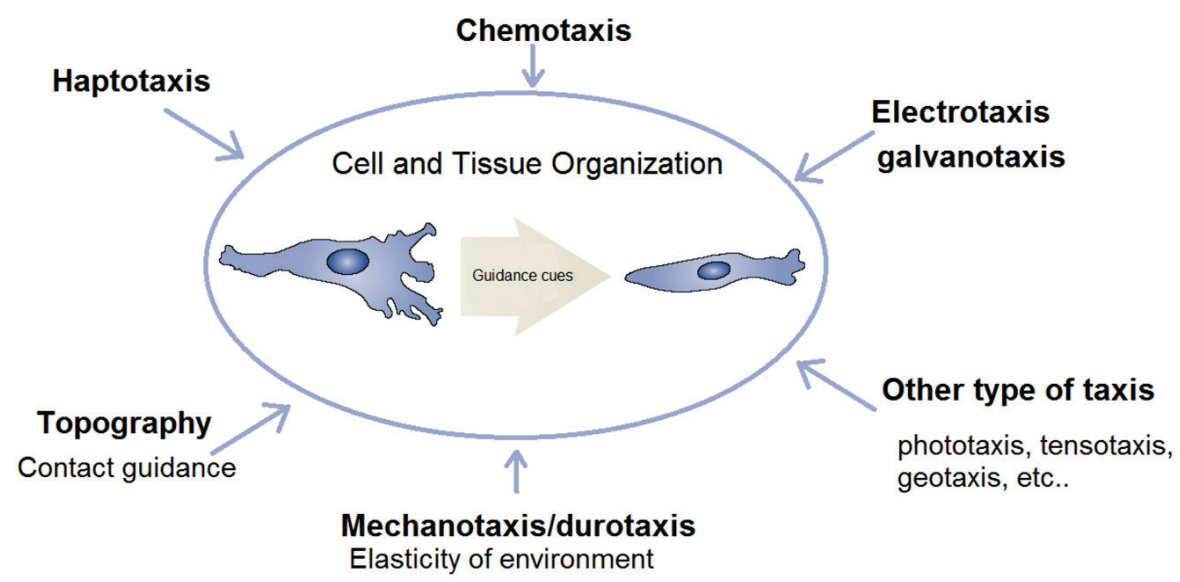

Figure 1. Directional control of cell migration can be induced by means of various taxis (moves to/away from stimulus) as schematized

It is widely renowned that cells perceive and explore the chemical and physical properties of a surrounding environment, gathering information at sites of ECM attachment and using them to activate specific signalling pathways within the cells $[9,10]$. The control of the cell environment by multiple physicochemical cues has therefore emerged as a key tenet to enable functionality, modulate response, and affect cell behaviour. The observation that cells are able to follow gradients of mechanical stiffness dates back to Lo et al. whom first reported that fibroblasts tend to move from softer to the stiffer regions of a matrix-coated substrate [11]. This phenomenon was coined as durotaxis after Latin durus (hard) and Greek taxis (regular arrangement). Since then, durotaxis or globally renowned as mechanotaxis, has been the object of numerous studies in the last two decades. However, although the effects of mechanical guidance underlying a broad diversity of functions on cells are becoming well known, experimental evidence is still scattered and mechanisms remain largely unknown. 


\subsection{THEORETICAL MECHANISMS OF DUROTAXIS}

The mechanisms underlying durotaxis, as abovementiond, are still poorly understood, but overall assume the involvement of active sensing and locomotion of the cell. Various theoretical suggestions on how the mechanism of durotaxis occurs have been proposed. One mechanosensing hypothesis theorises that cells move through the generation of traction by a "motor-clutch" mechanism with a centripetal flow of actin polymerization towards the centre of the cell which moves forward the cell's leading edge. Upon formation of every new pair of ligand-receptor bonds, a traction-bearing structure is either activated or anchored at the intracellular domain of the receptor, with focal adhesions on rigid ECM exerting high force and focal adhesions over soft ECM exerting low forces, as schematized in Figure 2. Sensing mediated by focal adhesions and actin-containing filopodia in front of the leading edge allow cells to determine physical characteristics of the substrate before moving and to avoid the formation of mature focal adhesions on soft substrates which would lead to backtracking of the cell. Stiffer substrates cause tension to develop at nascent adhesions, promoting the maturation of focal adhesions and allowing extension/spreading of the cell. On the other hand soft substrates hampers this increase of tension inhibiting maturation into focal adhesions, which then promotes the retraction of filopodia through myosin II-dependent contractile forces.

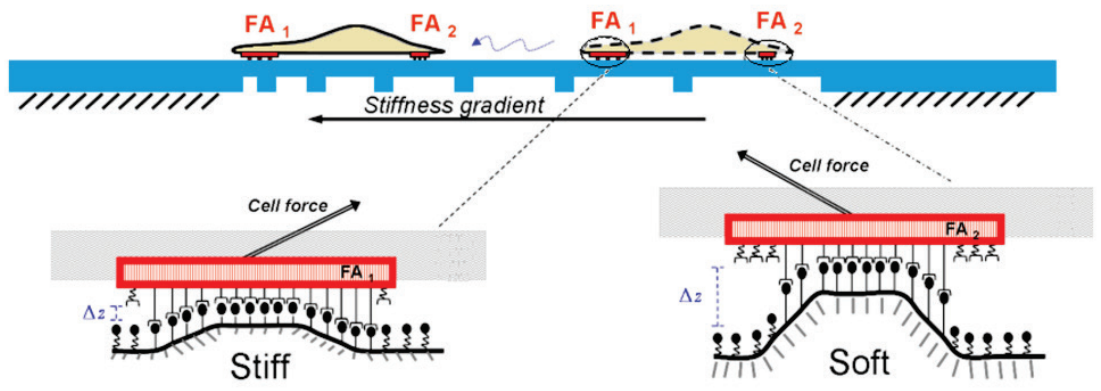

Figure 2. Schematization of focal ahdesions on stiffness gradient. On soft substrates cells do not develop abundant stress fibres and generate smaller forces, making adhesion sites on these regions less stable. In this way, a cell moving on a substrate with a stiffness gradient relocates towards the stiffer region.

Consequently, an asymmetry in traction force is generated leading to directed cell movement towards the more rigid substrate. Thus, forces and deformations, which occur through ECM linked proteins, modulate the dynamic flow of actin cytoskeleton during the durotactic movement [12]. 
Another theory proposed by Plotnikov and Waterman, hypothesises that cells attempt to maintain constant substrate deformation during migration $[13,14]$. This theory proposes that cell migration behaviour is mediated by the tugging motion on focal adhesions, migrating faster when the stiffness differences between the opposing ends of cells are greater [12]. In addition, cell migration on stiffer substrates involves higher forces to break cell to ECM bonds, which may explain the presence of pronounced stress fibers occurring in cells on stiff substrates such as plastic or glass.

An alternative theory has put forward that durotaxis is caused by the different levels of $\mathrm{Ca}^{2+}$ ions on the opposing extremities of the cell $[15,16]$. This theory sustains that the focal adhesions on higher stiffness support a more frequent contraction with consequential higher activation of stretch-mediated $\mathrm{Ca}^{2+}$ ion channels and cell migration towards regions of higher stiffness.

Although all these theoretical proposals are still under intense investigation, they remain the key basis theories for durotatic cell migration.

\subsection{DIVERSITY OF MECHANOTACTIC CELL BEHAVIOUR}

The mechanotactic behaviour of cells is cell-type dependant and context dependent [17-19]. Georges et al. reported that neurons and astrocytes react differently to the mechanical properties of the substrate. Astrocytes prefer stiff substrates, whereas they found that cortical neurons show more branched morphology on softer poly(acrylamide gels) (200 Pa vs $9000 \mathrm{~Pa}$ ) [20]. Balgude and co-workers also demonstrated that neurite extension of chick dorsal root ganglia (DRG) neurons was higher in softer gels [21]. According to Flanagan's group, the formation of neurite branches was enhanced by softer substrates [22]. Ulrich et al. observed that glioma cells cultured on fibronectin-coated polymeric ECM with varied but defined mechanical rigidity exhibited altered cell morphology and cytoskeletal organization. These authors showed that glioma cells cultured on softer substrates showed a decreased spreading area, disappearing stress fibres and focal adhesions. Interestingly, all evaluated glioma cell lines cultured on the softest substrates were rounded but viable with cortical rings of F-actin and punctuate vinculin-positive focal complexes, and with no indication of apoptosis [23]. Additionally studies reported by Leach et al., implied that there is an optimal substrate stiffness for neuronal cells that is between $10 \mathrm{~Pa}$ and $200 \mathrm{~Pa}$ [24].

Difference of cell functions, such as cell spreading, growth, and differentiation, were also observed to be modulated by the mechanic properties of the substrate. Pelham et al. reported that cells on flexible substrates showed reduced spreading and increased rates of motility compared to cells on rigid substrates [25]. Wang and his group observed cell proliferation to be increased on substrates of higher mechanical stiffness wheras the rate of apoptosis was increased on more flexible substrates [26]. Through investigating angiogenesis 
in vitro, Vailhe and co-workers demonstrated that the formation of capillary-like structures was influenced by the rigidity of the fibrin gels utilized [27]. Similarly Deroanne's group showed that cell differentiation was affected by the mechanical properties of the substrate. In fact decreasing the substrate rigidity increased the number of endothelial cells to switch to a tube-like pattern [28].

\subsection{INFLUENCE OF ECM STIFFNESS ON CELLS}

As abovementioned, cells connect and interact with their surrounding environment sensing and responding to its physical, mechanical environment, through cues from adjacent cells, external stress, and the ECM. The ECM is fundamental as it provides cells structural support as well as acting as an external cell-signalling region, being mostly composed of adhesive glycoproteins, proteoglycans, glycosaminoglycans and fibrous proteins that also incorporates biologically active molecules and growth factors. This process by which cells can sense rigidity of the ECM is referred to as mechanotransduction where the mechanical signals regulate and feedback the cytosketal reorganization and actomyosin contractility [29]. Cells, in fact, constantly survey the mechanical properties of their environment not only adhering to the matrix but also exerting a "pulling" force on the matrix substrate, which deforms the matrix in accordance with its elastic properties [30]. Forces that originate in the cytoskeleton are transmitted across the cell and eventually to ECM through specialized adhesion sites (focal adhesions). Focal adhesions are protein complexes connecting the cytoskeleton to the ECM at the sites of integrin binding. These complexes appear to act as plausible key signalling candidates in the translation of mechanical cues from the extracellular environment into biochemical signals inside the cell [31]. The integrin binds to different ECM specific ligands (fibronectin, collagen, laminin or vitronectin), hence, physically connecting the actin cytoskeleton to the ECM and influencing the downstream signalling events (including the activation of extracellular signal-regulated kinases (ERK), mitogen-activated protein kinases (MAP) and focal adhesion kinases (FAK)) which determines the final cell behaviour and fate [32,33]. Cells' responses to extracellular cues are therefore strongly linked to the myosin activity in the cytoskeleton, whereas the organization of the actin cytoskeleton is controlled by mechanical and geometrical properties of the surrounding matrix. Upon integrin activation, different cytoskeletal proteins like actin, talin and paxillin and signalling molecules such FAKs are connected and structured at the focal adhesion sites [34]. On stiff substrates which cannot deform under contraction, cells assume an unnatural flattened morphology, with increased proliferation rates and have also shown differences in cancer drug sensitivities [35,36]. This was explained by considering that stiffer substrates induce higher levels of expression of adhesion molecules (i.e. integrins), to improve adhesion [37]. Moreover, an increase in the synthesis of cellular structural proteins, such as 
actin was observed inducing different expression profiles [38]. On the other hand, cell spread area was shown to decrease with decreasing substrate stiffness and cells observed on soft substrates assumed a more rounded morphology with less focal adhesions and actin fibres, deforming in concurrence with the deformation of the surrounding soft matrix (Figure 3) [39].

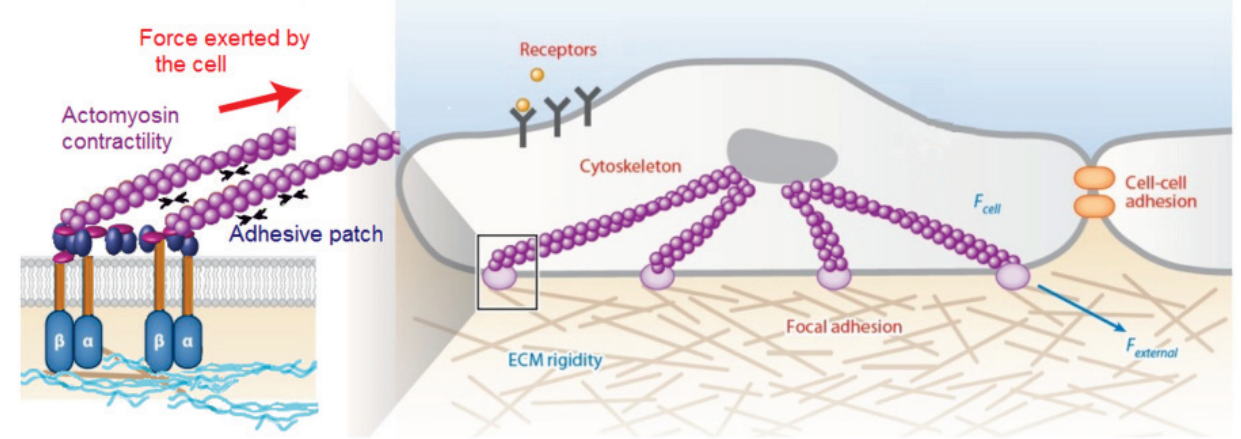

Figure 3. Cells response to extracellular cues is strongly linked to myosin activity in the cytoskeleton, whereas the organization of the actin cytoskeleton is controlled by mechanical and geometrical properties of the surrounding matrix. Cells adhere to the substrate through mechano-responsive molecular complexes, such as integrin-mediated adhesions, and locally and rapidly adapt to varying forces exerted at the cell-substrate interface.

Not all cell types display mechanosensing behaviour. Principally tissue forming cells including fibroblasts, mesenchymal stem cells, endothelial cells and epithelial cells were demonstrated to differ in behaviour with increased spreading and traction forces and decreased motility on stiffer substrates [16,18,39-41]. Fibroblasts and endothelial cells showed an intensification of spreading and a more increased assembly of their cytoskeleton into actin stress fibres and focal adhesions at $\sim 3 \mathrm{kPa}$. On the other hand pre-osteocytes re-organize their cytoskeleton and show increase spreading at $\sim 60 \mathrm{kPa}$ while neutrophils appeared to be insensitive to stiffness changes $[18,42]$. Stem cell differentiation was also shown to be influenced by stiffness of the ECM $[43,44]$. Klein investigated cell cycle progression regulated by ECM stiffness in endothelial cells through the activation of the small guanine triphosphatase (GTPase) RAC1 [45]. ECM stiffness was also showed to influence gene expression and cell fate. Engler and coworkers induced mesenchymal stem cells to differ in bone differentiation in stiff environments, whereas into adipocyte differentiation on soft substrates [40]. Analogous observations with skeletal muscle stem cells and with adult neural stem cells were reported 
$[46,47]$. Furthermore proteins of the hippo pathway were shown to be associated in the transcriptional effects of ECM stiffness and cytoskeletal organization, influencing the stiffness-dependent regulation of ECM gene expression and cell cycle progression [48].

As mechanosensitivity is associated to the cells' ability to rearrange adhesion ligands and to apply traction forces on the substrate, substrate mechanics and adhesiveness should be regarded as coupled variables. Peyton and Putnam reported a biphasic dependence of cell migration speed on ECM stiffness. In their study, the optimal stiffness at which cell migration speed is maximized was found to depend on the density of immobilized ECM ligands [49]. Investigating spreading of smooth muscle cells (SMCs) on collagen-coated poly(acrylamide gels), Engler's group showed that the cytoskeletal organization of muscle cells depends on the stiffness of the substrate on which they are grown with optimal substrate stiffness closest to that of relaxed muscle bundles [50]. They showed that matrix compliance and ligand density are highly coupled variables that determine mean cell responses. The same group stressed the importance of substrate stiffness on cellular motility for anchorage dependent cells [29]. Anchorage dependent, contractile cells show an increase in cellular adhesions and a more organized cytoskeleton with increasing stiffness of the substrate on which they are grown. This is explained considering the elasticity of their natural environment. In fact in vivo muscle and skin is much stiffer then the brain and the spinal cord and for this reason neurons prefer to migrate towards soft substrates, whereas muscle cells and fibroblasts favour stiffer substrates. Durotaxis therefore, occurs as a result of "positive feedback" of cells-substrate reinforcement as cell actively exerts forces on their ECM micro-environment through focal adhesions. Cells subject to durotaxis, can mediate signal transduction as ECM bound proteins or proteins linked to scaffolds connected to the ECM undergo conformational modifications as a result of changes to the ECM [51,52]. Scaffolding proteins allow in fact the linkage between integrins and actin cytoskeleton, which will form the ECM-integrin-cytoskeleton connections which develop to focal complexes [53]. In order for the focal complexes to mature to focal adhesions, and to connect to stress fibres, forces must be generated by the cell itself through its contractile machinery, but also from the surrounding environment [29]. More specifically, mechano-sensitive ion channels present near focal adhesion are activated as cells exert pulling forces [54-56]. This leads to an influx of extracellular calcium, increased level of acto-myosin contractility and reinforcement of the cell-substrate adhesions with clustering of focal adhesion complexes [57].

Concurrently, various proteins such as focal adhesion kinase, paxillin, vinculin and talin are recruited to reinforce the adhesion (Figure 4) [58,59]. As the local stiffness of the microenvironment increases, a solitary cell will consequently form a stronger adhesion on its substrate. Likewise, in the presence of a 
gradient of substrate rigidity, cell will have a tendency to form stronger adhesions on stiffer ECM regions.

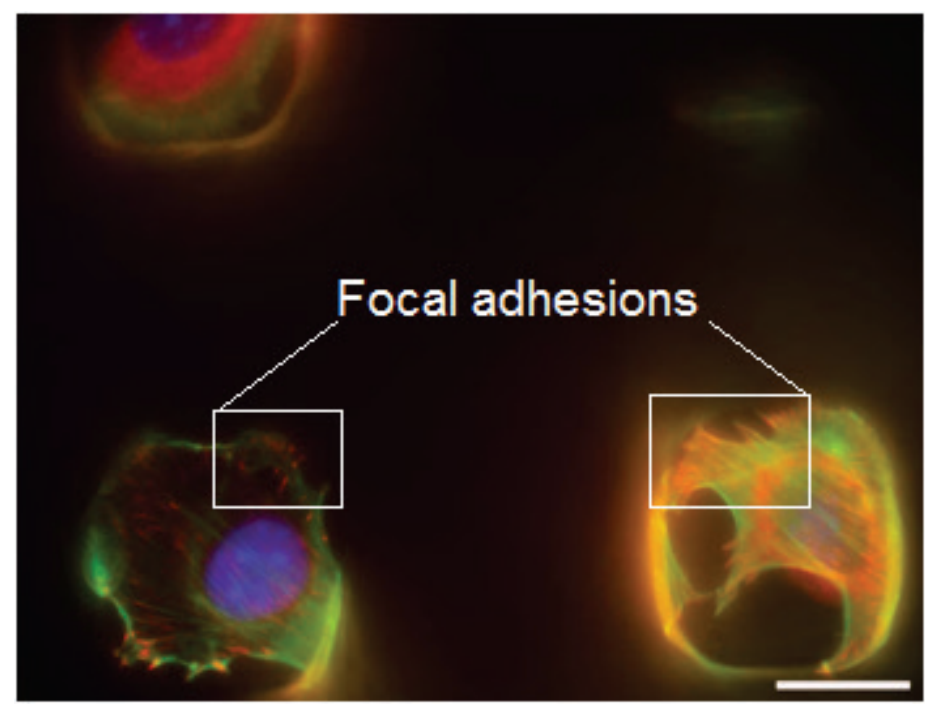

Figure 4. Fluorescent images of NIH 3T3, cultured for $24 \mathrm{~h}$, stained for vinculin in green, plated on square-patterned substrates and pillars in pits for $24 \mathrm{~h}$. DNA is blue and actin is red. The figures outline the stiff regions, caused by the presence of the square patterns underneath the membrane, whereas the regions surrounding the pillars are soft. The force is transmitted to the extracellular environment at cell-matrix contacts and actively generated by myosin molecular motors interacting with actin filaments. Scale bar: $20 \mu \mathrm{m}$.

Although many important advances have been acknowledged not much information about the diverse pathways activation and signal transduction is known. Hence, more investigations on the the role of the localization of integrin-modulated signal molecules in the regulation of cell differentiation by ECM elasticity is also an important target for future work.

\subsection{DIVERSITY OF MECHANICAL FEATURES OF THE SUBSTRATE}

Over the past years different methods have been used to create mechanical heterogeneities on substrates. So far, purely elastic poly(acrylic acid) (PAA) hydrogels as well as thin poly(acrylamide) gels have been widely used for mechanotaxis means [22,25,59]. Photocurable poly(acrylamide) gels are chemically cross-linked gels formed by the polymerization of acrylamide with a cross-linking agent, usually $N, N^{\prime}$-methylenebisacrylamide. These gels are 
widely used as a compliant substrate for mechanotaxis although the unpolymerized acrylamide monomer is highly toxic and often results in loss of cell viability. Poly(dimethylsiloxane) (PDMS) commonly used in biomedical applications and in membrane technology for its biocompatibility, mechanical compliance, chemical inertness, and remarkable flexibility has also been used for prototyping substrates for mechanotaxis means [60-63]. Moreover these elastomers may have an elastic moduli able to effectively cover the entire range of soft tissue elastic moduli from approximately $1 \mathrm{kPa}$ to $>1 \mathrm{MP}$ [64-66]. This is advantageous as at the tissue-scale range the stiffness of cell microenvironment displays high variation within the body. Between different tissues, ECM rigidity often varies over several orders of magnitude, e.g., brain (260-490 Pa), liver (640 Pa), kidney (2.5 kPa), skeletal muscle $(12-100 \mathrm{kPa})$ and cartilage (950 kPa) [67]. Tissue variation can also be triggered by pathological factors such as malignant tumours, which are stiffer than the surrounding healthy tissue [68].

Differences of elasticity have been with various approaches. Ohashi and colleagues showed that cells develop stress fibres in response to nonuniform strain and development of these stress fibres eventually leads to a shift in the location of the cell nucleus [69]. The monotonically increasing strain gradient was obtained by embedding glass within a silicone elastomer and stretching the substrate. The lowest strain region, close to the glass-elastomer border showed no signs of cellular elongation or orientation; however cells within the region of high strain alignined perpendicular in direction of stretch. They also showed a strain dependent formation of stress fibres exists which led to cell remodelling due to the strain gradient. Similarly cells also tend to have more pronounced alignment if microgrooves in the substrate are perpendicular to stretch than if they are parallel to the direction of stretch [70]. Loesberg work showed that mechanical loading played a secondary role in fibroblast phenotype modulation while substrate topography was the primary influence.

Kuntanawat and co-workers simply modified the thickness of the film, altering the apparent elasticity of the substrate by variation in height of a thin poly(acrylamide) gel [71]. The thicker the gel, the less influence has the mechanical property of the (hard) substrate, thus the softer the surface showing that cells migrated along the gradient. A method reported used to generate patterns of elasticity is through the control of the polymerization sites through photomasks or laser beams. Nemir et al. used a base polymer and soaked the solid gel with a solution containing a different polymer precursor. The precursor penetrated the network of the gel and was polymerized via UV-curing at determined positions with the aid of a photomask. The unreacted precursor was then removed, leaving areas composed only of the base polymer [72]. A similar approach was used by Hahn which used a two-photon absorption (TPA) photolithography to pattern bioactivity bioactive features into optically transparent, photoactive materials by diffusing a second precursor inside a base polymer and removal of the unreacted pre-polymer 
[73]. Kloxin and co-workers demonstrated to be able to create gradients in crosslinking density with a predictable tuning of the gel structure in real-time with low-intensity, long-wavelength UV light. They used a UV-degradable cross-linker, undoing the network at the positions affected by the laser [74]. A different strategy was followed by Chou and colleagues. The pattern was created by filling the channels of a topographically patterned sample with a second polymer of different composition [75].

However one must take into account that the chemistry of the precursors is different and may be sufficient to form a chemical gradient which can override the mechanical one. Patterns of elasticity can be prepared by coating solid structures with a relatively soft film. To date, the work of Cortese and coworkers, has showed that a single material has been a without changes in other major surface and/or bulk properties to be able to influence cell behaviour $[6,76]$. PDMS substrates were developed where the elastic modulus could be easily and independently tuned to mimic soft tissues over a three order-of-magnitude range. The pattern was created by utilizing the contrast between air chambers and the geometry of the underlying bulk substrate. Topographic patterns of PDMS with desired geometry were fabricated and covered with a thin elastic film, creating air pockets between the patterned structures (Figure 5).
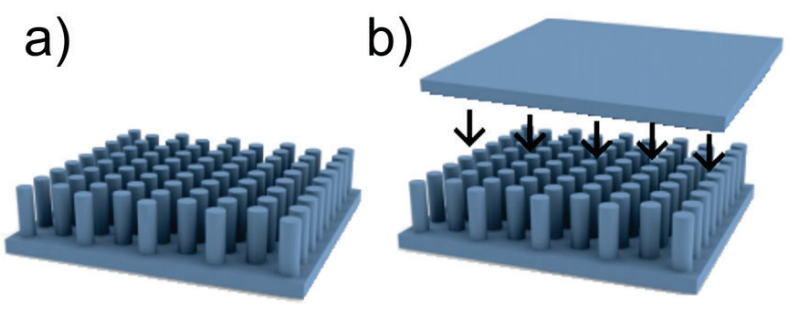

C)

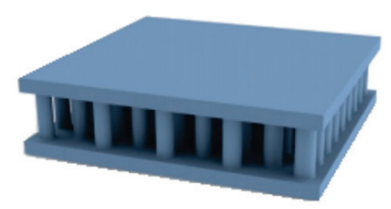

Figure 5. Schematization of the fabrication process of the thin membrane on the substrate. a) A PDMS patterned substrate was created by pouring a pre-polymer of PDMS on a pre-patterned SU8 master substrate b) Bonding of substrate and a thin PDMS membrane, $c$ ) the final substrate with membrane for mechanotaxis purpose was obtained.

The thin membrane PDMS film was studied in various mass to mass ratios to create low stiffness gels. Moreover by varying the mechanical rigidity of the film the elasticity could be finely tuned as well as cell behaviour [6]. To investigate the conditions to induce mechanotaxis, different PDMS membrane thin films with different surface elasticity conditions were prepared by changing the base to curing ratios i.e. the degrees of crosslinking and dilution with heptane $[6,76]$. The embedded structures affect the elasticity of determined areas of the substrate, while others remain unaffected (either no 
solid structure is located underneath or the film thickness is high enough to hide the effect of the structure). When cells move towards the high Young's modulus area of the substratum, the cells would extend their lamellipodia and move directly onto the rigid part of the substrate. In contrast, when cells migrate from stiffer substrates (i.e. lines, Figure 6) the cells would move up and down the mechanical interface and cross over onto the stiff regions without residing on the softer side. Thus, mechanical interactions between a cell and its underlying substratum play a crucial role in modulating cell motility.

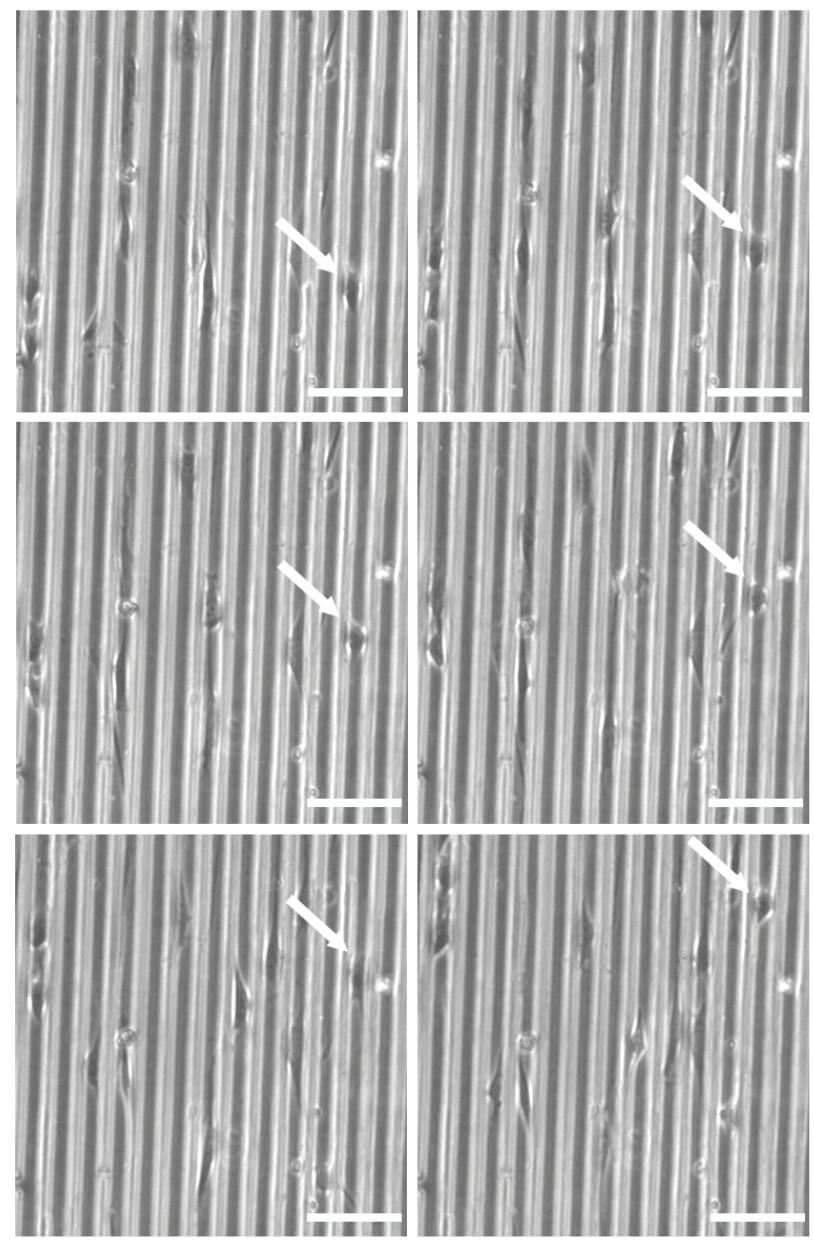

Figure 6. Time-lapse phase contrast images of human fibroblasts cells moving on the membrane. The darker lines are the patterned grooves underneath the membrane. In this sequence of images, cells feel and respond to the substrate clearly moving along the ridges of the lines on the harder regions. Attention has been focused with the arrow on a particular cell, moving rapidly towards the top. Images were obtained approximately $1 \mathrm{~h}$ after seeding cells on the substrate, and the interval between each image was about $30 \mathrm{~min}$. Scale bar: $100 \mu \mathrm{m}$. Figure is adapted from ref. [6]. 


\subsection{CELL'S RESPONSE TO FORCE}

Rigidity sensing and durotaxis responses can be quantified by measuring traction forces while cells are simultaneously interacting with substrates of different rigidities. The concept of durotaxis is even more complicated by the cell's response to force. Not only the cell detects externally applied force but it must respond appropriately to this force by actively exerting force on the substrate by deformation of the substrate. This process as a positive feedback loop, requires cell migration to mediate the function of durotaxis both actively and passively simultaneously. An approach to measure these forces has been developed using substrates composed of flexible arrays of micropost arrays [77-81]. Micropost arrays present a powerful topographic technology to measure he cellular traction forces on the substrate. Specifically, when cells are seeded onto arrays of identical circular microposts, they spread over several posts (Figure 7) and can physically pull at the tops of the posts, resulting in visible displacements. The deflection of each post provides a direct measurement of the local force exerted by the attached cells independently of the forces acting on the neighbouring posts. The pillars simply act as independent springs and the linear theory of elasticity gives their deflection (Figure 7).
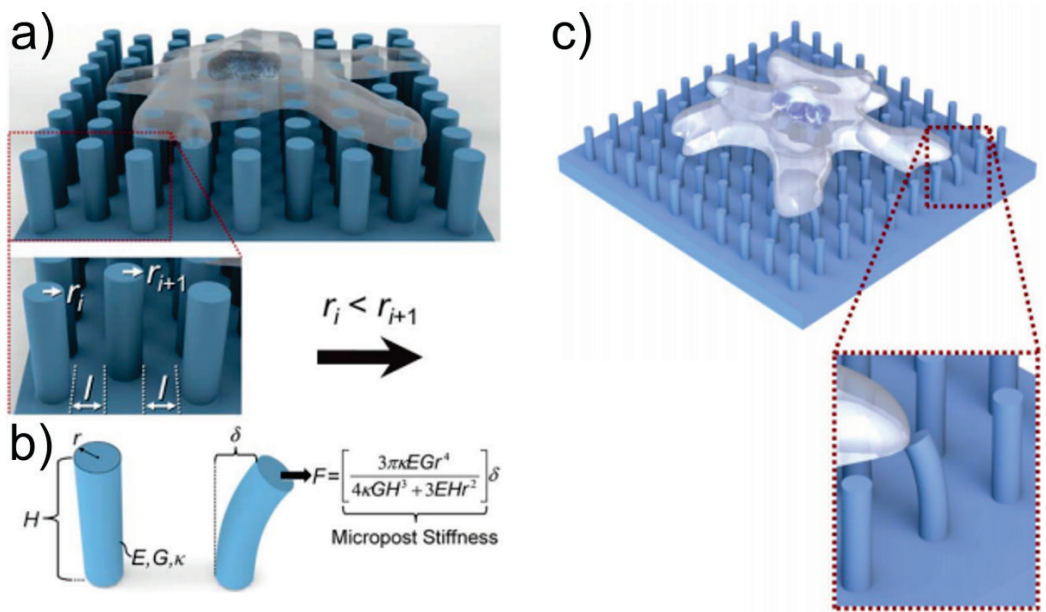

Figure 7. Micropost array. (a) Schematic illustration of a cell seeded on a microtopographic stiffness gradient with an enlarged view of individual microposts with increasing radii $(r)$ and equivalent interpost spacing $(I)$. (b) Micropost cantilever model. The linear stiffness at the top of the micropost is derived from its geometric and material properties, including the Young's modulus $(E)$, shear modulus $(G)$, shear coefficient $(k)$, micropost height $(H)$, and micropost radius $(r)$. A force applied at the top of the micropost $(F)$, parallel to the substrate, will result in a displacement at the top of the micropost $(\delta)$. c) Physically pull of the tops of the microposts by the cell, resulting in visible displacement of the post. Figure is adapted from ref. [80]. 
For a cylinder of radius $r$ and length $H$ bent by the application of a force $F$, it leads to the following formula:

$$
F=k_{\text {bend }} \delta=\left(\frac{3 \pi k E G r^{4}}{4 k G H^{3}+3 E H r^{2}}\right) \delta
$$

where $E, G, k, k_{\text {bend }}$ and $\delta$ are, respectively, the Young modulus, the shear modulus, the shear coefficient, the spring constant and the deflection of the post. A very interesting work was presented by Sochol and co-workers. Their micropost arrays of varying anisotropy showed an optimize unidirectional control of cell migration through dual axis durotaxis cues restricting movement in the lateral direction in addition to promoting migration in the direction of increasing micropost stiffness. Additionally, higher gradient strength was found to enhance this directional response [82].
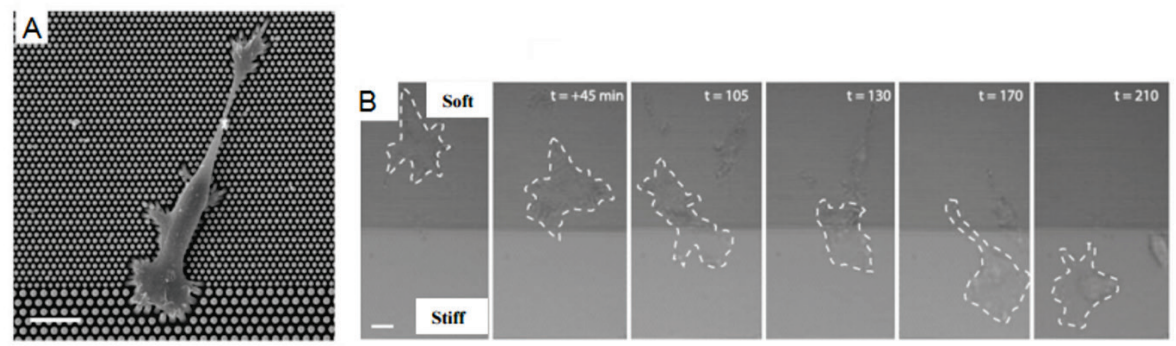

Figure 8. Scanning electron microscopy image of cells spreading on micropillar surface of two different diameters, where micropillars with larger diameter are stiffer (A). Cells positioned at the interface of the stiff and soft micropillar interface preferentially migrated and resided onto the stiffer surface (B). Figure is adapted from ref. [83].

Trichet's group demonstrated that when fibroblast cells are grown on array of anisotropic micro-pillars presenting soft and stiff micropillars, these cells have a tendency to migrate towards and reside on the stiff region (Figure 8) [83]. These studies suggest that the cytoskeleton, specifically actomyosin contractility, is crucial for rigidity sensing during durotaxis.

In conclusion, the ability to measure traction forces while cells interact with substrates of different rigidities is a key step to unravelling the mechanism underlying durotaxis. Micropillared arrays enable high control over positioning and geometry using microfabrication and provide a powerful tool for investigating the cellular response to mechanical properties of the substrate. However, more studies are indispensable to investigate the presence of cell-cell interaction, as cells integrate polarizing mechanotransductive cues from not only the substrate but also from neighboring cells. It has been shown in fact that collective migration in epithelial sheets enhanced transmission of mechanical force signals transmitted through cell-cell adhesions [84]. 
Therefore cells combine mechanical cues from both the substrate, as in the form of rigidity, and contacting cells associated to physical forces exerted at cell-cell contacts.

\subsection{OVERRIDING ROLE OF CHEMOTAXIS OR MECHANOTAXIS?}

An important experimental question that should be investigated is: does chemotaxis override durotaxis during cell migration? Although the molecular mechanisms to detect external stimuli of durotaxis are not identical to that of chemotaxis, there might exist common downstream signalling pathways that drive polarization and migration [52]. Similarly to chemotaxis, cells undergoing durotaxis can sense physical forces and transduce them into biochemical signals. What distinguishes the two phenomena is that durotaxis does not require biochemical transduction to direct cellular motion. Knowledge of the parameters of chemotaxis and durotaxis would therefore help in the design of ECM scaffold stiffness.

Understanding the integrated mechanotactic responses of cells to the microenvironment is necessary in order to predict and control the behaviours of cells for therapeutic applications. In particular, understanding how to regulate the cell shape and functions, controlling the signals coming from ECM and the external forces are the primal tasks in tissue engineering as well as in development of functional biomaterials. Then, providing insight into what, where and when a cell senses ECM cues and activates its biochemical responses is fundamental to understand how such cues can be incorporated into new, three-dimensional scaffolds to treat diseases. Besides, insight of cell motility per se has important consequences for improving clinical treatments as therapeutic interventions that either interferes with mechanotransductive signalling or mechanical remodelling. These considerations are especially important for example in the development of anti-cancer therapies, which might be able to target aspects that are dysregulated in this kind of disease. Nonetheless, the role of mechanotaxis and its controlling mechanisms are still under extensive study.

An important step in research for cellular response to mechanical gradients is the design of a substrate that can control directional movement of cells maintaining unvaried the physical and chemical properties to avoid undesirable responses. Thus, to fully understand the role of mechanics on cell function it would be ideal to tailor the elastic modulus without altering other material properties such as surface energy, chemistry and roughness. This type of engineered substrate is useful for studying separately the chemical and mechanical stimuli contributing to the time-dependent migration of different cell types. 


\section{ACKNOWLEDGEMENTS}

This study was supported by the Italian Association for Cancer Research (AIRC) through the grant MFAG n. 16803.

\section{REFERENCES}

1. R.R. Kay, P. Langridge, D. Traynor, O. Hoeller. Nat. Rev. Mol. Cell. Biol. 9 (2008) 455.

2. F. Pagani, R.C. Paolicelli, E. Murana, B. Cortese, S. Di Angelantonio, E. Zurolo, E. Guiducci, T.A. Ferreira, S. Garofalo, M. Catalano, G.D’Alessandro, A. Porzia, G. Peruzzi, F. Mainiero, C. Limatola, C.T. Gross, D. Ragozzino. Front. Cell. Neurosci. 9 (2015)111.

3. B. Cortese, I.E. Palamà, S.D'Amone, G. Gigli. Integr. Biol. 6 (2014) 817.

4. B. Cortese, M.O. Riehle, S.D’Amone, G. Gigli. J. Coll. Inter. Sci. 394 (2013) 582.

5. B. Cortese, C. Piliego, I. Viola, S. D’Amone, R. Cingolani, G. Gigli. Langmuir 25 (2009) 7025.

6. B. Cortese, G. Gigli, M.O. Riehle. Adv. Funct. Mater. 19 (2009) 2961.

7. M.J. Dalby, N. Gadegaard, R.O.C. Oreffo. Nat. Mat. 13 (2014) 558.

8. M.J Dalby, N. Gadegaard, R. Tare, A. Andar, M.O Riehle, P. Herzyk, C.D.W Wilkinson, R.O.C Oreffo. Nat. Mat. 6 (2007) 997.

9. A.D. Bershadsky, N.Q.Balaban, B. Geiger. Annu. Rev. Cell Dev. Biol. 19 (2003) 677.

10. B. Geiger, J.P. Spatz, A.D. Bershadsky. Nat. Rev. Mol. Cell. Biol. 10 (2009) 21.

11. C.M. Lo, H.B. Wang, M. Dembo, Y.L. Wang. Biophys. J. 79 (2000) 1.

12. C.E. Chan, D.J. Odde. Science 322 (2008) 1687.

13. S.V. Plotnikov, A.M. Pasapera, B. Sabass, C.M. Waterman. Cell 151 (2012) 1.

14. S.V. Plotnikov, C.M. Waterman. Curr. Opin. Cell Biol. 25 (2013) 619.

15. T. Mammoto, A. Mammoto, D.E. Ingber. Annu. Rev. Cell Dev. Biol. 29 (2013) 27.

16. T. Kobayashi, M. Sokabe. Curr. Opin. Cell Biol. 22 (2010) 669.

17. R.J. Young, P.A. Lovell. Introduction to Polymers, $3^{\text {rd }}$ Ed., CRC Press, USA, 2011, p. 469.

18. T. Yeung, P.C. Georges, L.A. Flanagan, B. Marg, M. Ortiz, M. Funaki, N. Zahir, W. Ming, V. Weaver, P.A. Janmey. Cell Motil. Cytoskeleton 60 (2005) 24.

19. S. Nemir, J.L. West. Ann. Biomed. Eng. 38 (2010) 2.

20. P.C. Georges, W.J. Miller, D.F. Meaney, E.S. Sawyer, P.A. Janmey. Biophys. J. 90 (2006) 3012.

21. A.P. Balgude, X. Yu, A. Szymanski, R.V. Bellamkonda. Biomaterials 22 (2001) 77.

22. L.A. Flanagan, Y.E. Ju, B. Marg, M. Osterfield, P.A. Janmey. NeuroReport 13 (2002) 2411.

23. T.A. Ulrich, E.M. de Juan Pardo, S. Kumar. Cancer Res. 69 (2009) 4167.

24. J.B. Leach, X.Q. Brown, J.G. Jacot, P.A. Dimilla, J.Y. Wong. J. Neural. Eng. 4 (2007) 26.

25. R.J. Pelham, Y.L. Wang. Proc. Natl. Acad. Sci. 94 (1997) 13661.

26. H.B. Wang, M. Dembo, Y.L. Wang. Am. J. Physiol. Cell Physiol. 279 (2000) C1345.

27. B. Vailhé, X. Ronot, P. Tracqui, Y. Usson, L. Tranqui. In Vitro Cell. Dev. Biol. Anim. 33 (1997) 763. 
28. C.F. Deroanne, C.M. Lapiere, B.V. Nusgens. Cardiovasc. Res. 49 (2001) 647.

29. D.E. Discher, P. Janmey, Y.L. Wang. Science 310 (2005) 1139.

30. C.S. Chen. J. Cell. Sci. 121 (2008) 3285.

31. D.E. Ingber. Faseb J. 20 (2006) 811.

32. E. Ruoslahti. Adv. Cancer Res. 76 (1999) 1.

33. M.E Hemler, R.R. Lobb. Curr. Opin. Hematol. 2 (1995) 61.

34. D. Riveline, E. Zamir, N.Q. Balaban, U.S. Schwarz, T. Ishizaki, S. Narumiya, Z. Kam, B. Geiger, A.D. Bershadsky. J. Cell Biol. 153 (2001) 1175.

35. L. David, V. Dulong, D. Le Cerf, L. Cazin, M. Lamacz, J.P. Vannier. Acta Biomater. 4 (2008) 256.

36. J.L Horning, S.K. Sahoo, S. Vijayaraghavalu, S. Dimitrijevic, J.K. Vasir, T.K. Jain, A.K. Panda, V. Labhasetwar. Mol Pharm. 5(2008) 849.

37. J. Du, X. Chen, X. Liang, G. Zhang, J. Xu, L. He, Q. Zhan, X.-Q. Feng, S. Chien, C. Yan. Proc. Natl. Acad. Sci. U.S.A. 108 (2011) 9466.

38. P.A. Janmey, R.T. Miller. J. Cell. Sci. 124 (2011) 9.

39. M. Dembo, Y.L. Wang. Biophys. J. 76 (1999)2307.

40. A.J. Engler, S. Sen, H.L. Sweeney, D.E. Discher. Cell 126 (2006) 677.

41. $\quad$ R.J. Pelham, Y.L. Wang. Biol. Bull. 194 (1998) 348.

42. H.J. Kong, T.R Polte, E. Alsberg, D.J. Mooney. Proc. Natl Acad. Sci. U.S.A. 102 (2005) 4300.

43. F. Watt, W. Huck. Nat. Rev. Mol. Cell Biol. 14 (2013) 467.

44. P. Lu, V. Weaver, Z. Werb. J. Cell Biol. 196 (2012) 395.

45. D.E.A. Klein, L. Yin, D. Kothapalli, P. Castagnino, F.J. Byfield, T. Xu, I. Levental, E. Hawthorne, P.A. Janmey, R.K. Assoian. Curr. Biol. 19 (2009) 1511.

46. P.M. Gilbert, K.L. Havenstrite, K.E. Magnusson, A. Sacco, N.A. Leonardi, P. Kraft, N.K. Nguyen, S. Thrun, M.P. Lutolf, H.M. Blau. Science 329 (2010) 1078.

47. K. Saha, A.J. Keung, E.F. Irwin, Y. Li, L Little, D.V. Schaffer, K. E. Healy. Biophys. J. 95 (2008) 4426.

48. S. Dupont, L. Morsut, M. Aragona, E. Enzo, S. Giulitti,M. Cordenonsi, F. Zanconato, J. Le Digabel, M. Forcato, S. Bicciato, N. Elvassore, S. Piccolo, Nature 474 (2011) 179.

49. $\quad$ S.R. Peyton, A.J. Putnam. J. Cell. Physiol. 204 (2005) 198.

50. A.J. Engler, M.A. Griffin, S. Sen, C.G. Bönnemann, H. Lee Sweeney, D.E. Discher, J. Cell Biol. 166 (2004) 877.

51. D.E. Discher, P. Janmey, Y.L. Wang. Science 310 (2005) 1139.

52. P. Roca-Cusachs, T. Iskratsch, M.P. Sheetz. J. Cell Sci. 125 (2012) 3025.

53. P. Roca-Cusachs, R. Sunyer, X. Trepat. Curr. Opin. Cell Biol. 25 (2013) 543.

54. B. Geiger, A. Bershadsky, R. Pankov, K.M. Yamada. Nat. Rev. Mol. Cell Biol. 2 (2001) 793.

55. H. Wolfenson, A. Bershadsky, Y.I. Henis, B. Geiger. J. Cell. Sci. 124 (2011) 1425.

56. F. Sachs. Mol. Cell. Biochem. 104 (1991) 57.

57. J. Arnadóttir, M. Chalfie. Ann. Rev. Biophys. 39 (2010) 111.

58. C. Grashoff, B.D. Hoffman, M.D. Brenner, R. Zhou, M. Parsons, M.T. Yang, M.A. McLean, S.G. Sligar, C.S. Chen, T. Ha, M.A. Schwartz. Nature 466 (2010) 263.

59. A. Engler, L.e Bacakova, C. Newman, A. Hategan, M. Griffin, D. Discher, Biophys. J. 86 (2004) 617.

60. J.C. McDonald, G.M. Whitesides. Acc. Chem. Res. 35 (2002) 7.

61. C.F. Adams, A.J. Paul. J. Crustacean Biol. 19 (1999) 1. 
62. Y. Zhao, C.C. Lim, D.B. Sawyer, R. Liao, X. Zhang. J. Micromech. Microeng. 15 (2005) 9.

63. A. Gadre, M. Kastantin, S. Li, R. Ghodssi, Proc. Int. Semiconductor Device Research Symp. Washington DC, USA, 2001, p. 186.

64. X.Q. Brown, K. Ookawa, J.Y. Wong. Biomaterials 26 (2005) 3123.

65. E. Gutierrez, A. Groisman. PLoS One 6 (2011) e25534.

66. P.Y. Wang, W.B. Tsai, N.H. Voelcker. Acta Biomater. 8 (2012) 519.

67. I. Levental, P.C. Georges, P.A. Janmey. Soft Matter. 3 (2007) 299.

68. S. Kumar, V.M. Weaver. Cancer Metastasis Rev. 28 (2009) 113.

69. T. Ohashi, M. Masuda, T. Matsumoto, M. Sato. Clin. Hemorheol. Microcirc. 37(2007) 37.

70. W.A. Loesberg, X.F. Walboomers, J.J.W.A. van Loon, J.A. Jansen. J. Biomed. Mat. Res. 75A (2005) 723.

71. P. Kuntanawat, C. Wilkinson, M. Riehle. Comp. Biochem. Physiol. Part A Mol. Integr. Physiol. 146 (2007) S192.

72. $\quad$ S. Nemir, H.N. Hayenga, J.L. West. Biotechnol. Bioeng. 105 (2010) 636.

73. M.S. Hahn, J.S. Miller, J.L. West. Adv. Mater. 18 (2006) 2679.

74. A.M. Kloxin, M.W. Tibbitt, A.M. Kasko, J.A. Fairbairn, K.S. Anseth. Adv. Mater. 22 (2010) 61.

75. $\quad$ S.-Y. Chou, C.-M. Cheng, P.R. LeDuc, Biomaterials 30 (2009) 3136.

76. I. E. Palamà, S. D’Amone, A. M. L. Coluccia, M. Biasiucci, G. Gigli, Integr. Biol. 4 (2012) 228.

77. F. Binamé, G. Pawlak, P. Roux, U. Hibner. Mol. Biosyst. 6 (2010) 648.

78. W.C. Wei, H.H. Lin, M.R. Shen, M.J. Tang. Am. J. Physiol. Cell Physiol. 295 (2008) C1579.

79. N.J. Sniadecki, C.S. Chen. Methods Cell Biol. - Cell Mechanics 83 (2007) 313.

80. L. Tan, J. Tien, D.M. Pirone, D.S. Gray, K. Bhadriraju, C.S. Chen. Proc. Natl. Acad. Sci. U.S.A. 100 (2003) 1484.

81. I.K. Lin, Y.-M. Liao, Y. Liu, K.-S. Ou, K.-S. Chen, X. Zhang. Appl. Phys. Lett. 93 (2008) 251907.

82. R.D. Sochol, A.T. Higa, R. Janairo, S. Li, L. Lin. Soft Matter. 7 (2011) 4606.

83. L. Trichet, J. Le Digabel, R.J. Hawkins, S.R.K. Vedula, M. Gupta, C. Ribrault, P. Hersen, R. Voituriez, B. Ladoux. Proc. Natl. Acad. Sci. U.S.A. 109 (2012) 6933.

84. M.R. Ng, A. Besser, G. Danuser, J.S. Brugge. J. Cell Biol. 199 (2012) 545. 